Musées, Patrimoine et Culture scientifiques et techniques

$153 \mid 2014$

mai-juin 2014

\title{
Les collections des muséums d'Histoire naturelle en chiffres
}

Patrice Charon

\section{OpenEdition \\ Journals}

Édition électronique

URL : http://journals.openedition.org/ocim/1367

DOI : $10.4000 /$ ocim. 1367

ISSN : 2108-646X

Éditeur

OCIM

Édition imprimée

Date de publication : 25 juin 2014

ISSN : 0994-1908

Référence électronique

Patrice Charon, «Les collections des muséums d'Histoire naturelle en chiffres », La Lettre de I'OCIM [En ligne], 153 | 2014, mis en ligne le 25 juin 2016, consulté le 20 avril 2019. URL : http:// journals.openedition.org/ocim/1367; DOI : 10.4000/ocim.1367 


\section{Les collections des muséums $d^{\prime}$ Histoire naturelle en chiffres}

\section{Patrice Charon}

Chargé d'études, OPCST-OCIM

En 2012, l'Observatoire de l'OCIM débutait une enquête d'envergure sur les muséums d'Histoire naturelle en France, via la plateforme OCIM-muséums mise en place à cet effet et déjà expérimentée lors d'une première édition portant sur les données 2010. Dans le cadre de cette enquête, beaucoup plus détaillée que la précédente, a été engagée une exploration fine des collections des muséums, concernant les types de disciplines abritées (familles et composantes) ainsi que leur importance numérique.
Ont été recensés 68 établissements français en activité (ouverts ou fermés temporairement) pour l'année 2011, 3 n'ayant pas participé au dispositif. Plus précisément, il s'agit de muséums d'Histoire naturelle stricto sensu, et de musées disposant de collections naturalistes valorisées au plan scientifique et/ou culturel.

Les collections des muséums sont par nature très diversifiées : Sciences naturelles (Botanique, Géologie, Paléontologie, Zoologie), Sciences humaines (Archéologie,

\begin{tabular}{|c|c|c|c|c|c|c|c|c|c|c|}
\hline \multirow[t]{2}{*}{ Commune } & \multirow{2}{*}{ Botanique } & \multirow{2}{*}{ Géologie } & \multirow{2}{*}{ Paléontologie } & \multicolumn{3}{|c|}{ Zoologie } & \multirow{2}{*}{ Archéologie } & \multirow{2}{*}{ Ethnologie } & \multirow{2}{*}{ TOTAL } & \multirow{2}{*}{$\begin{array}{l}\text { Fourchette } \\
\text { de volume }\end{array}$} \\
\hline & & & & Total & Vertébrés & Invertébrés & & & & \\
\hline DIJON & 250200 & 11500 & 50000 & 2054039 & 4039 & 2050000 & & 300 & 2366039 & Plus de 1 Million \\
\hline LYON & 2800 & 15000 & 170000 & 1604200 & 32700 & 1571500 & 30000 & 25000 & 1847000 & Plus de 1 Million \\
\hline $\mathrm{NICE}$ & 450000 & 100000 & 350000 & 378000 & 18000 & 360000 & & & 1278000 & Plus de 1 Million \\
\hline AIX-EN-PROVENCE & 231550 & 14186 & 69530 & 131760 & 3176 & 128575 & 15194 & 471 & 462691 & 100000 - 1 million \\
\hline ANGERS & 352150 & 8000 & 51020 & 85150 & 4950 & 80200 & 40000 & 116 & 536436 & 100000 - 1 million \\
\hline AUTUN & 302550 & 150000 & 260000 & 102190 & 11190 & 91000 & 10000 & & 824740 & $100000-1$ million \\
\hline AUXERRE & 10000 & 11000 & 40000 & 41950 & 1700 & 40250 & & & 102950 & $100000-1$ million \\
\hline AVIGNON & 451076 & 50000 & 275950 & 117815 & 6314 & 111501 & & 280 & 895121 & $100000-1$ million \\
\hline BESANÇON & 529000 & & 100000 & 181700 & 1445 & 180000 & & 600 & 811300 & $100000-1$ million \\
\hline BORDEAUX & & 15000 & 515070 & 342140 & 15590 & 326550 & & & 872210 & 100000 - 1 million \\
\hline BOURGES & 75137 & 7702 & 22352 & 121055 & 10055 & 111000 & 1000 & 188 & 227434 & 100000 - 1 million \\
\hline CHAMBÉRY & 90500 & 1500 & 10020 & 31155 & 855 & 30300 & & & 133175 & $100000-1$ million \\
\hline CLERMONT-FERRAND & 170969 & 70000 & 10000 & 377946 & 6946 & 371000 & & & 628915 & $100000-1$ million \\
\hline GAP** & 60040 & 20000 & 5000 & 15570 & 10070 & 5500 & 7000 & 480 & 108090 & $100000-1$ million \\
\hline GRENOBLE & 500000 & 16650 & 43150 & 314000 & 14000 & 300000 & 4005 & 1647 & 879452 & $100000-1$ million \\
\hline LA ROCHELLE & 80000 & 6000 & 10000 & 43682 & 11662 & 32020 & 8594 & 10030 & 158306 & $100000-1$ million \\
\hline LAVAL & 33000 & 22000 & 66000 & 11240 & 3240 & 8000 & & & 132240 & $100000-1$ million \\
\hline LE HAVRE & 24200 & 20000 & 90000 & 24500 & 2500 & 22000 & 100000 & 900 & 259600 & 100000 - 1 million \\
\hline LE MANS & 55000 & 5000 & 20000 & 87400 & 2300 & 85100 & & & 167400 & 100000 - 1 million \\
\hline LILLE & & 56400 & 120000 & 201650 & 11600 & 190050 & 2000 & 13000 & 393050 & 100000 - 1 million \\
\hline MARSEILLE & 65180 & 8250 & 79600 & 86007 & 10907 & 75100 & 3000 & 2550 & 244587 & $100000-1$ million \\
\hline MONTBÉLIARD * * & 91009 & 6530 & 94500 & 41760 & 4830 & 36930 & 200000 & & 433799 & $100000-1$ million \\
\hline NANCY & 53 & & 52950 & 136700 & 18700 & 118000 & 252 & 165 & 190120 & $100000-1$ million \\
\hline NANTES & 210150 & 46000 & 70000 & 151930 & 30550 & 121375 & 1600 & NR & 479680 & 100000 - 1 million \\
\hline NIIMES & 109780 & 25000 & 5000 & 18640 & 11370 & 7269 & 80000 & 2000 & 240420 & $100000-1$ million \\
\hline NIORT** & 70000 & 1600 & 9500 & 24840 & 2010 & 22830 & 1537 & 2846 & 110323 & $100000-1$ million \\
\hline
\end{tabular}


Points de vue complémentaires

\begin{tabular}{|c|c|c|c|c|c|c|c|c|c|c|}
\hline \multirow[t]{2}{*}{ Commune } & \multirow{2}{*}{ Botanique } & \multirow{2}{*}{ Géologie } & \multirow{2}{*}{ Paléontologie } & \multicolumn{3}{|c|}{ Zoologie } & \multirow{2}{*}{ Archéologie } & \multirow{2}{*}{ Ethnologie } & \multirow{2}{*}{ TOTAL } & \multirow{2}{*}{$\begin{array}{l}\text { Fourchette } \\
\text { de volume }\end{array}$} \\
\hline & & & & Total & Vertébrés & Invertébrés & & & & \\
\hline ORLÉANS & 20400 & 11600 & 4450 & 322580 & 2580 & 320000 & 45000 & 986 & 405016 & $100000-1$ million \\
\hline ROUEN & 100200 & 7498 & 250000 & 120890 & 14685 & 106200 & 27367 & 2500 & 508455 & $100000-1$ million \\
\hline SAINT-QUENTIN & 500 & & & 200175 & 95 & 200080 & & & 200675 & $100000-1$ million \\
\hline STRASBOURG & & & 1308 & 971033 & 55640 & 915393 & & 20 & 972361 & $100000-1$ million \\
\hline TOULOUSE & 3094 & 10000 & 15000 & 463480 & 33314 & 430160 & 20000 & 4600 & 516174 & $100000-1$ million \\
\hline TROYES** & 75000 & 23170 & 28028 & 360000 & 10000 & 350000 & & & 486198 & $100000-1$ million \\
\hline ANNECY** & 11600 & 2900 & 3600 & 2850 & 1150 & 1700 & 20000 & 20000 & 60950 & $10000-100000$ \\
\hline AURILLAC & 10150 & 2600 & 3200 & 1770 & 612 & 1157 & & & 17720 & $10000-100000$ \\
\hline BAYONNE & 4075 & 2000 & 100 & 6815 & 1785 & 5030 & NR & 52 & 13042 & $10000-100000$ \\
\hline BÉZIERS & 10000 & NR & NR & 5 & NR & $N R$ & NR & NR & 10005 & $10000-100000$ \\
\hline BLOIS & 3050 & 4100 & 3600 & 21000 & 1000 & 20000 & & & 31750 & $10000-100000$ \\
\hline CHARTRES & 15050 & 7500 & 7500 & 7000 & 1000 & 6000 & 50000 & & 87050 & $10000-100000$ \\
\hline CHÂTEAUDUN** & 570 & 1000 & 50 & 5602 & 3602 & 2000 & 2530 & 850 & 10602 & $10000-100000$ \\
\hline $\begin{array}{l}\text { CHERBOURG- } \\
\text { OCTEVILLE }\end{array}$ & & 2000 & 2400 & 9722 & 1722 & 8000 & 4000 & 5000 & 23122 & $10000-100000$ \\
\hline CHILHAC & & & 20000 & & & & & & 20000 & $10000-100000$ \\
\hline GAILLAC & 5000 & 8000 & 31000 & 19723 & 2362 & 17361 & 515 & & 64238 & $10000-100000$ \\
\hline GRAY & 7725 & 2337 & 15444 & 6382 & 926 & 5456 & 50 & & 31938 & $10000-100000$ \\
\hline LODÈVE * * & & 318 & 1572 & & & & 15000 & & 16890 & $10000-100000$ \\
\hline MONTAUBAN & 5400 & 1700 & 6140 & 10113 & 4113 & 6000 & 71500 & 51 & 94904 & $10000-100000$ \\
\hline PERPIGNAN & 9500 & 3000 & 3000 & 82720 & 1618 & 81095 & & 140 & 98360 & $10000-100000$ \\
\hline SAINTTDENIS & 200 & 1820 & 400 & 66600 & 24576 & 42024 & & 51 & 69071 & $10000-100000$ \\
\hline $\begin{array}{l}\text { SAVIGNÉ-SUR-LA- } \\
\text { THAN }\end{array}$ & & & 12526 & & & & & 710 & 13236 & $10000-100000$ \\
\hline $\begin{array}{l}\text { SEMUR- } \\
\text { EN-AUXOIS** }\end{array}$ & $N R$ & 12000 & 12000 & 20500 & 200 & 20300 & 1300 & 200 & 46000 & $10000-100000$ \\
\hline TOULON & 36020 & 4250 & 12300 & 31646 & 1446 & 30200 & 900 & 91 & 85207 & $10000-100000$ \\
\hline BARCELONNETTE* * & 52 & 190 & 38 & 2750 & 2490 & 260 & NR & NR & 3030 & $1000-10000$ \\
\hline COLMAR & 200 & 5000 & & & & & & 550 & 5750 & $1000-10000$ \\
\hline DECAZEVILLE & & 1500 & 1500 & & & & & & 3000 & $1000-10000$ \\
\hline ELBEUF & NR & NR & NR & NR & NR & NR & NR & NR & & $1000-10000$ \\
\hline $\begin{array}{l}\text { LA CHAIZE- } \\
\text { LE-VICOMTE }\end{array}$ & & & & 2080 & 2005 & 71 & & & 2080 & $1000-10000$ \\
\hline MARCIAC & & & 500 & 4650 & 2650 & 2000 & & & 5150 & $1000-10000$ \\
\hline MENAT & & & 10000 & & & & & & 10000 & $1000-10000$ \\
\hline MULHOUSE & & 850 & 2000 & 30 & 30 & & 100 & & 2980 & $1000-10000$ \\
\hline MURAT & & & & 8800 & 800 & 8000 & & & 8800 & $1000-10000$ \\
\hline TOURS & 4 & 280 & 2410 & 1941 & 361 & 1580 & & & 4635 & $1000-10000$ \\
\hline VILLERS-SUR-MER & & & NR & 76 & 76 & & & & 76 & $1000-10000$ \\
\hline CAEN & & & & 610 & 610 & & & & 610 & $100-1000$ \\
\hline LE BLANC & & & & 819 & 819 & & NR & NR & 819 & $100-1000$ \\
\hline LE PRADET & & 1000 & & & & & & & 1000 & $100-1000$ \\
\hline VACHÈRES & & & 161 & & & & 650 & 10 & 821 & $100-1000$ \\
\hline TOTAL & 4532134 & 803931 & 3039869 & 9479381 & 422966 & 9056117 & 763094 & 96384 & 18714793 & \\
\hline
\end{tabular}

* L'ensemble des musées recensés disposent de l'appellation « Musée de France » à l'exception du Pradet et de Murat.

** || s'agit ici d'autres musées (dont anciens « musées-mixtes ") n'ayant à répondre que pour la partie $\mathrm{HN}$.

NR : Non répondant 
Ethnologie-Anthropologie), autres collections scientifiques (Astronomie, Médecine, Pharmacie, Physiquechimie, Histoire des sciences, Ouvrages, Instruments) et parfois collections culturelles (Art, Histoire...). Toutes ces collections comportent elles-mêmes de nombreuses sous-disciplines, en particulier les Sciences naturelles aux composantes variées ${ }^{(1)}$.

Ces collections rassemblent aujourd'hui de très grandes quantités d'objets mais leur comptage demeure une mission délicate compte tenu de leurs natures variées : taille ; matière ; intégrité (individus complets ou partiels, fragmentaires, morcelés, populations) ; lots ; ensembles... et de la grande diversité des types de conditionnements des lots : tubes, bocaux, boites, tiroirs, cartons, herbiers... Ceci fait de la démarche de dénombrement un défi en lui-même pour les muséums, sachant que chacun d'entre eux a développé sa propre manière de compter ses collections.

Après un travail de catégorisation des collections et en étroite collaboration avec la Conférence Permanente des Muséums de France (CPMF), l'Observatoire de l'OCIM a mis en place un cadre de comptage commun en phase avec la pratique des professionnels des collections : avec d'un côté la mention du nombre d'objets et/ou de spécimens inventoriés, du nombre total estimé ; de l'autre la mention des unités et/ou des lots, cela pour chaque discipline représentée. Une campagne de recueil encadrée de plusieurs mois a ainsi permis de donner lieu à une quantification de ce patrimoine, voulue au plus proche de la réalité en tenant compte des différentes contraintes de dénombrement des collections, tout en intégrant une marge d'erreur liée à la contenance aléatoire des lots. Voici la synthèse des résultats, s'appuyant sur les 65 répondants (hors le premier chiffre général).

On dénombre en France 68 muséums, dont 66 ayant l'appellation "Musée de France ", sur un total de 1220 musées de France ${ }^{(2)}$, représentant près de $6 \%$ de l'ensemble.

Par volumes de collections, les muséums se répartissent ainsi :

\begin{tabular}{|l|r|r|}
\hline Fourchette de volume & $\begin{array}{l}\text { Nombre } \\
\text { d'établissements }\end{array}$ & Part du Total \\
\hline Plus de 1 million d'objets & 3 & $4,60 \%$ \\
\hline Entre 100000 et 1 million d'objets & 29 & $44,60 \%$ \\
\hline Entre 10000 et 100000 objets & 18 & $27,69 \%$ \\
\hline Entre 1000 et 10000 objets & 11 & $16,92 \%$ \\
\hline Entre 100 et 1000 objets & 4 & $6,15 \%$ \\
\hline Total & 65 & $100 \%$ \\
\hline
\end{tabular}

Hormis le groupe des plus de 1 million d'objets, le nombre d'établissements croît proportionnellement au nombre d'objets détenus. Les établissements détenant entre 100000 et 1 million d'objets, au nombre de 29 (soit 44,6\% de l'ensemble), sont le groupe le plus représenté.

Plus largement et au plan des « Musées de France », on observe que sur les 50 musées de plus de 100000 objets ${ }^{(3)}, 32$ sont des muséums, soit $64 \%$, faisant apparaître que ces derniers sont parmi les plus gros détenteurs d'objets en France.

Parallèlement, les 68 muséums abritent un nombre total de 18,7 millions d'objets, ce qui représente $52 \%$, soit plus de la moitié, des 36 millions d'objets ${ }^{(4)}$ détenus au total par les musées de France.

Par discipline, les collections se répartissent ainsi :

\begin{tabular}{|c|c|c|c|}
\hline \multicolumn{2}{|c|}{ 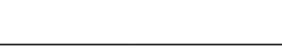 } & Nombre d'objets & \% TOTAL \\
\hline \multicolumn{2}{|c|}{ Botanique } & 4500000 & $24,1 \%$ \\
\hline \multicolumn{2}{|l|}{ Géologie } & 800000 & $4,3 \%$ \\
\hline \multicolumn{2}{|c|}{ Paléontologie } & 3000000 & $16,1 \%$ \\
\hline \multirow{3}{*}{ Zoologie } & Total & 9480000 & $50,9 \%$ \\
\hline & Vertébrés & 420000 & $2,3 \%$ \\
\hline & Invertébrés & 9060000 & $48,6 \%$ \\
\hline \multicolumn{2}{|c|}{ Archéologie } & 760000 & $4,1 \%$ \\
\hline \multicolumn{2}{|c|}{ Ethnologie } & 100000 & $0,5 \%$ \\
\hline \multicolumn{2}{|c|}{ TOTAL (estimé) } & 18700000 & $100,0 \%$ \\
\hline
\end{tabular}

Les disciplines les plus volumineuses sont les Invertébrés, la Botanique puis la Paléontologie. On observe de fortes disparités entre les établissements selon les disciplines et notamment des spécialités ou présences marquées.

\begin{tabular}{|l|l|}
\hline \multicolumn{2}{|l|}{ Les plus gros détenteurs par discipline } \\
\hline Botanique & Besançon, Grenoble, Avignon, Nice, Angers \\
\hline Géologie & Autun, Nice, Clermont-Ferrand, Lille, Avignon \\
\hline Paléontologie & Bordeaux, Nice, Avignon, Autun, Rouen \\
\hline Zoo-vertébrés & Strasbourg, Toulouse, Lyon, Nantes, Saint-Denis \\
\hline Zoo-invertébrés & Dijon, Lyon, Strasbourg, Toulouse, Clermont-Ferrand \\
\hline Archéologie & Montbéliard, Le Havre, Nîmes, Montauban, Chartres \\
\hline Ethnologie & Lyon, Annecy, Lille, La Rochelle, Cherbourg-Octeville \\
\hline
\end{tabular}

La répartition des collections dans les établissements s’avère très inégale :

- 8 établissements (soit 12,3\%) abritent plus de la moitié des collections ; 


\section{Points de vue complémentaires}

- 17 établissements (soit 26,2 \%) abritent près des 4/5 des collections ;

- 23 établissements (soit 35,4\%) abritent environ $1 \%$ des collections.

Les muséums d'Histoire naturelle occupent en termes d'importance numérique des collections une place de premier plan parmi les musées français. Leurs collections totalisent d'importantes quantités d'objets rendant la mission du récolement décennal particulièrement complexe pour la plupart d'entre eux. Ceci est accentué par les caractéristiques du patrimoine naturaliste qui requiert une méthodologie de récolement spécifique et où, similairement aux démarches de dénombrement et d'inventaire scientifique, la question de l'unité de comptage demeure centrale.

\section{Notes}

(1) Voir l'arborescence des collections sur la Plateforme OCIM-Muséums http://observatoire.ocim.fr/inventaire-museums

(2) 1159 musées de France recensés en décembre 2013 (donnée MCC 2013).

(3) 50 musées de France de plus de 100000 objets ont été mentionnés par le Service des musées de France à l'occasion de la deuxième journée nationale sur le récolement décennal des musées de France organisée à Paris le 12 décembre 2013

(4) Donnée SMF- DGPP-MCC 2013 (Deuxième journée nationale sur le récolement décennal des musées de France - Paris - 12 décembre 2013). 